\title{
Commentary on: Physalin B attenuates liver fibrosis via suppressing LAP2 $\alpha$-HDAC1 mediated deacetylation of GLI1 and hepatic stellate cell activation
}

\author{
Ralf Weiskirchen ${ }^{1}$ \\ ${ }^{1}$ RWTH Aachen University
}

April 22, 2021

- Invited Commentary -

Commentary on:

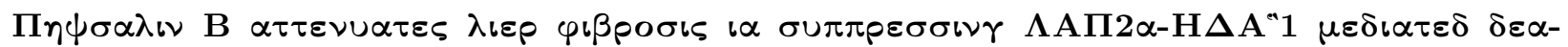

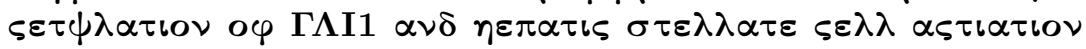

\section{Ralf Weiskirchen}

Institute of Molecular Pathobiochemistry, Experimental Gene Therapy and Clinical Chemistry (IFMPEGKC), RWTH University Hospital Aachen, Aachen, Germany; Email: rweiskirchen@ukaachen.de

Word count: 1193

Liver fibrosis is an excessive scarring process resulting in progressive disruption of the normal tissue architecture and impaired organ function. It comprises many different etiologies and its consequences present a substantial medical and economic burden worldwide (Weiskirchen et al., 2018). Mechanistically, fibrosis is initiated by parenchymal cell destruction resulting in tissue damage that is associated with an inflammatory response. This in turn provokes the local activation of mesenchymal cells which have the capacity to produce extracellular matrix compounds such as collagens. Most important in this process are hepatic stellate cells (HSCs) that are pericytes in the perisinusoidal space of the liver. Upon activation, these cells lose their quiescent phenotype and transit in a process called transdifferentiation into proliferative fibrogenic $\alpha$-smooth muscle positive ( $\alpha$-SMA) myofibroblasts. These are the central cellular drivers of hepatic fibrogenesis in experimental and human liver injury. Although the cellular and molecular mechanisms of hepatic fibrosis including important pro-inflammatory and pro-fibrogenic soluble mediators (chemokines, cytokines) and signaling pathways are identified, there exist no specific and effective antifibrotic pharmacological therapies.

Beside synthetic drugs, complementary and alternative medicine is presently in the focus of basic and clinical research. In particular, a number of herbal remedies and active ingredients thereof show promising effects against hepatic fibrosis either experimentally in cell culture, in pre-clinical animal models, or even in the first clinical trials (Weiskirchen 2015). These compounds counteract the intercellular production or activity of reactive oxygen species, prevent hepatic infiltration of circulating inflammatory acting blood cells, or interfere with relevant signaling pathways or mediators involved in the production or turnover of extracellular matrix.

The present study by Zhu and colleagues investigated the antifibrotic activities of Physalin B (PB) in two models of hepatic fibrosis, in primary cultures of mouse HSC, and in the well-established immortalized human HSC cell line LX-2.

$\mathrm{PB}$ possesses a unique 13,14-seco -16,24-cyclo -steroidal skeleton and an $\mathrm{H}$-ring with a $\mathrm{C}_{14}$-O- $\mathrm{C}_{27}$ bond 
forming an intriguing cage-shaped structure (Fang et al., 2003). It is a main active physalin of Physalis angulata $L$. belonging to the nightshade family (Solanaceae ). When the authors applied PB in mice that were either simultaneously subjected to repeated applications of carbon tetrachloride or to a bile duct surgery that are both established experimental models of hepatic fibrosis, the seco-steroid ameliorated hepatic fibrosis as assessed by overall reduced hepatic collagen deposition, lowered blood markers of liver damage (alanine aminotransferase, aspartate aminotransferase), and diminished expression of fibrogenic markers (collagen type I, tissue inhibitor of metalloproteinase 1 (TIMP1), $\alpha$-SMA, and transforming growth factor- $\beta$ (TGF- $\beta$ )). In cultured HSCs, PB decreased $\alpha$-SMA expression during spontaneous activation and transdifferentiation. Moreover, PB was effective to blunt TGF- $\beta$-induced activity of a luciferase-based COL1A1 reporter in human HSC line LX-2. Similarly, PB repressed glioma-associated oncogene 1 (GLI1) mRNA expression and activity of a GLI1-dependent reporter construct in primary mouse HSCs and in the immortalized human HSC line LX-2, while the expression of GLI2 and GLI3 were unaffected. Interestingly, the drug effectively blocked GLI1-induced expression of collagen and $\alpha$-SMA in cultured HSCs, most likely by hindering GLI1 nuclear localization that in turn resulted in reduced mRNA expression of typical GLI1 target genes such as Hedgehog Interacting Protein, Cyclin D, Cyclin E , and c-myc . Moreover, PB induced GLI1 acetylation by blocking complex formation between Lamina-associated polypeptide $2 \alpha$ (LAP2 $\alpha$ ) and histone deacetylase 1 (HDAC1) which normally promotes GLI1 deacetylation. The same stimulatory effect on GLI1 acetylation was induced by the HDAC inhibitor vorinostat confirming the protective effect of the LAP2 $\alpha /$ HDAC1 complex on GLI1 deacetylation.

The finding that PB treatment mitigated liver fibrosis viainhibiting the interaction between LAP2 $\alpha$ and HDAC1 that provokes increased acetylation and nuclear translocation of GLI1 is novel. In a related study, the same authors have recently shown that PB ameliorates experimental non-alcoholic steatohepatitis (NASH) in mice by stimulating autophagy and p62-Keap1-Nrf2 anti-oxidative signaling, suggesting that this drug has also some general anti-inflammatory and hepatoprotective activities (Zhang et al., 2021). In line, in a more previous report PB was considered to inhibit NO production by lipopolysaccharide- or interferon- $\gamma$-activated macrophages and to protect mice against a lethal lipopolysaccharide challenge (Soares et al., 2003). Thus, $\mathrm{PB}$ has the potential to counteract a broad range of biological processes that contribute to the pathogenesis of hepatic fibrosis. In particular, the inhibition of collagen, $\alpha$-SMA, TGF- $\beta$, and TIMP1 provides evidence that PB exerts anti-fibrogenic properties in the liver by inhibiting HSC activation. During hepatic fibrosis, the upregulation of these pro-fibrogenic marker genes is a hallmark indicating activation and transdifferentiation of quiescent HSCs into proliferative, profibrogenic and extracellular matrix-producing myofibroblasts.

The study provides novel molecular insights into the biological activity of PB and in principal opens new avenues for the implementation of novel anti-fibrotic therapies. However, the study has still some limitations. In particular, the authors investigated the anti-fibrotic activities only in in vitro and in vivo models of ongoing hepatic fibrogenesis showing that the drug efficiently impacts disease initiation and progression. It will be essential in future studies to prove the curative effectiveness in suitable models. Moreover, previous biological evaluation of different physalins in a panel of ten human and murine cancer cell lines demonstrated that PB has a broad cytotoxic activity towards most of the cell lines tested with a half-maximal inhibitory concentration $\left(\mathrm{IC}_{50}\right)$ in the range of $0.6 \mu \mathrm{M}-2.7 \mu \mathrm{M}$ (Fang et al., 2003). In the present study, Zhu and colleagues determined an $\mathrm{IC}_{50}$ of $5 \mu \mathrm{M}$ for the immortalized human HSC line LX-2 and used in their animal experiments concentrations of $1-5 \mathrm{mg} / \mathrm{kg}$ body weight that were applied intraperitoneally for 14 (bile duct ligation model) or 28 consecutive days (carbon tetrachloride model). The concentrations used in luciferase reporter assays and cell apoptosis testing were in the range of $0.25-1.0 \mu \mathrm{M}$, concentrations that might be already toxic to other cell types. Moreover, inhibition of GLI1 by PB might have several unwanted side effects. GLI1 is a transcriptional effector of the Hedgehog $(\mathrm{Hh})$ signaling pathway playing key roles in the development and homeostasis of many organs and tissues. Transcriptional suppression, aberrant posttranslational modification such as hyper-acetylation, or untargeted trafficking of this zinc finger protein will ultimately influence gene transcription and transcriptional output of the Hh pathway. Moreover, the HhGli pathway is relevant for stimulating cell divisions in undifferentiated progenitor cells and for committing cells to a specific fate. 
Effective anti-fibrotic therapies should include the attenuation of excessive matrix synthesis and deposition, the replacement of dysfunctional liver tissue, and the restoration of the original tissue architecture. Liver regeneration and remodeling is a well-coordinated process requiring increased cell proliferation and differentiation. Genetic cell lineage-tracing approaches have shown that damaged hepatocytes and biliary epithelial cells are renewed from common liver progenitor cells and liver repair requires the activity of immune cells. Therefore, it will be of fundamental importance to test if the beneficial effects of PD in preventing extracellular matrix synthesis interfere with the dynamic and adaptive changes necessary in during the process of reconstitution of functional liver tissue.

In sum, the current work has identified a new drug candidate effective in experimental hepatic fibrosis. It will now be necessary to perform well-designed safety and efficacy studies. Future work will show if the observed experimental anti-fibrotic activities of thisseco -steroid can be factually translated to the clinic.

Funding : The author is supported by grants from the German Research Foundation (projects WE2554/131 and W2554/15-1). The funder had no role in the design of this comment or in the decision to publish it.

Conflict of interest statement : The author has nothing to declare.

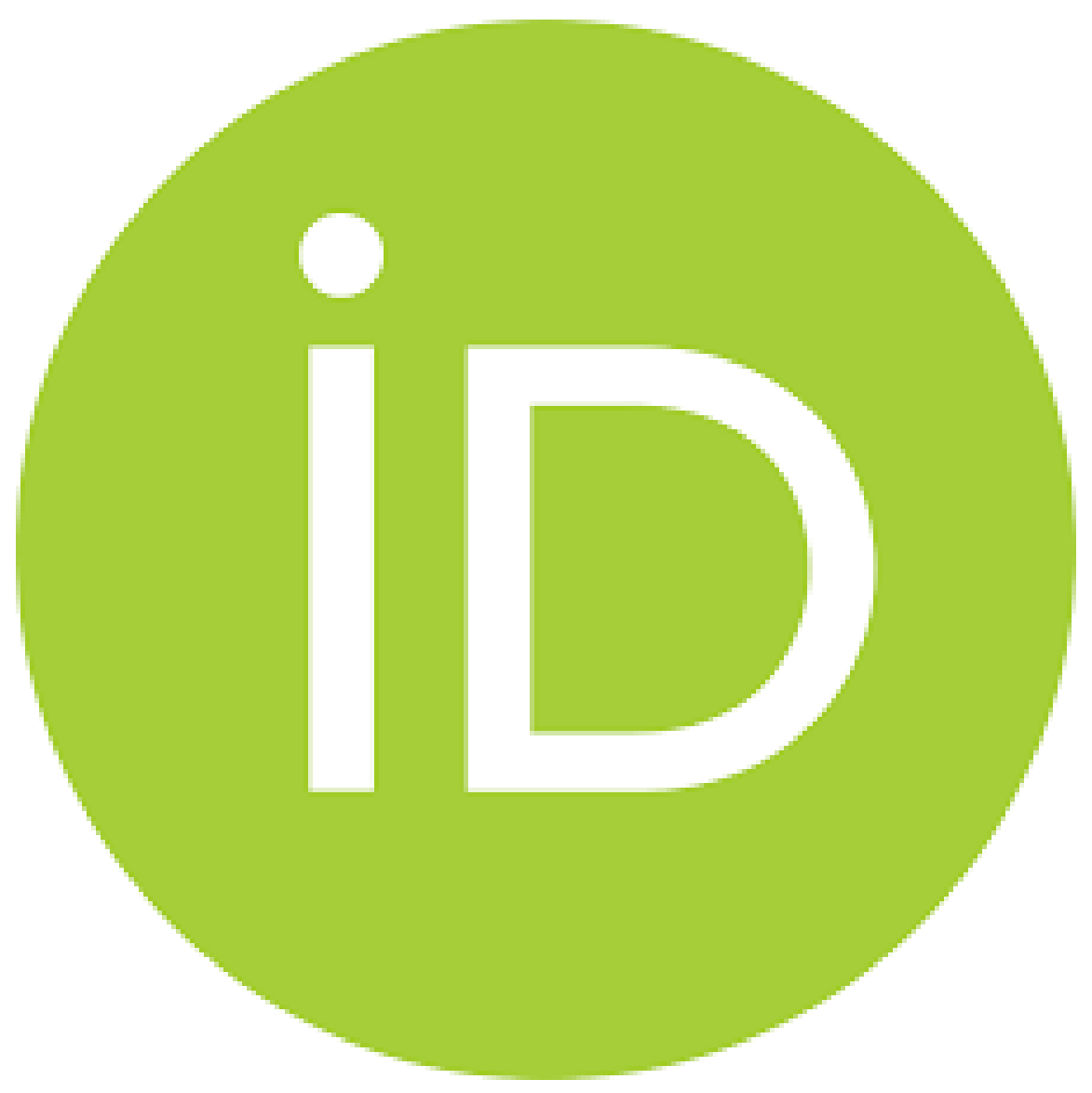

\section{ORCID}

Ralf Weiskirchen https://orcid.org/0000-0003-3888-0931

\section{References}


Fang, L., Chai, H. B., Castillo, J. J., Soejarto, D. D., Farnsworth, N. R., Cordell, G. A., Pezzuto, J. M., \& Kinghorn, A. D. (2003). Cytotoxic constituents of Brachistus stramoniifolius . Phytother Res .17 (5), 520-523. doi: $10.1002 /$ ptr.1315

Soares, M. B., Bellintani, M. C., Ribeiro, I. M., Tomassini, T. C., \& Ribeiro dos Santos, R. (2003). Inhibition of macrophage activation and lipopolysaccaride-induced death by seco-steroids purified fromPhysalis angulata L . Eur J Pharmacol . 459 (1), 107-112. doi: 10.1016/s0014-2999(02)02829-7

Weiskirchen, R. (2016). Hepatoprotective and anti-fibrotic agents: It's time to take the next step. Front Pharmacol . 6 :303. doi: 10.3389/fphar.2015.00303

Weiskirchen, R., Weiskirchen, S., \& Tacke F. (2018). Recent advances in understanding liver fibrosis: bridging basic science and individualized treatment concepts. F1000Res . 7 :F1000 Faculty Rev-921. doi: 10.12688/f1000research.14841.1

Zhang, M. H., Li, J., Zhu, X. Y., Zhang, Y. Q., Ye, S. T., Leng, Y. R., Yang, T., Zhang, H., \& Kong, L. Y. (2021). Physalin B ameliorates nonalcoholic steatohepatitis by stimulating autophagy and NRF2 activation mediated improvement in oxidative stress. Free Radic Biol Med . 164, 1-12. doi: 10.1016/j.freeradbiomed.2020.12.020 